\title{
Seizures and Epilepsy: An Overview for Neuroscientists
}

\author{
Carl E. Stafstrom ${ }^{1}$ and Lionel Carmant ${ }^{2}$ \\ ${ }^{1}$ Division of Pediatric Neurology, Departments of Neurology and Pediatrics, Johns Hopkins University School \\ of Medicine, Baltimore, Maryland 21287 \\ ${ }^{2}$ Division of Neurology, Department of Pediatrics, Sainte-Justine Hospital, Universite Montreal, Montreal, \\ Quebec H3T 1C5, Canada \\ Correspondence: cstafst1@jhmi.edu; lionel.carmant@umontreal.ca
}

\begin{abstract}
Epilepsy is one of the most common and disabling neurologic conditions, yet we have an incomplete understanding of the detailed pathophysiology and, thus, treatment rationale for much of epilepsy. This article reviews the clinical aspects of seizures and epilepsy with the goal of providing neuroscientists an introduction to aspects that might be amenable to scientific investigation. Seizures and epilepsy are defined, diagnostic methods are reviewed, various clinical syndromes are discussed, and aspects of differential diagnosis, treatment, and prognosis are considered to enable neuroscientists to formulate basic and translational research questions.
\end{abstract}

Tas his article provides an overview of seizures and epilepsy for neuroscientists. We focus on broad concepts, rather than clinical details, and raise questions related to mechanisms, epileptogenesis, and therapeutic approaches that might generate interest among basic researchers. Further information about differential diagnosis, drug doses, and clinical management are available from numerous resources (Engel and Pedley 2008; Duchowny et al. 2012; Engel 2013).

We first define seizures and epilepsy and summarize their classification, pathophysiology, and genetics. Diagnostic methods are then considered, including the importance of an accurate historical description of an event suspected to be a seizure and the appropriate use of ancillary/confirmative tests, such as electroencephalogram (EEG), neuroimaging, and genetic studies. These modalities enable the clini- cian to differentiate epilepsy from numerous clinical conditions that mimic seizures, but have a nonepileptic pathophysiological basis. Examples of epilepsy syndromes are then described, selected based on their frequency in the population or because they embody scientific questions that warrant elucidation. Finally, we provide an overview of treatment options and prognosis, including a consideration of conditions that accompany epilepsy (comorbidities) and complicate the daily lives of people with epilepsy. Subsequent articles in this collection explore the scientific basis of many of the clinical concepts introduced here.

\section{DEFINITIONS AND EPIDEMIOLOGY}

A "seizure" is a paroxysmal alteration of neurologic function caused by the excessive, hyper-

Editors: Gregory L. Holmes and Jeffrey L. Noebels

Additional Perspectives on Epilepsy: The Biology of a Spectrum Disorder available at www.perspectivesinmedicine.org

Copyright (C) 2015 Cold Spring Harbor Laboratory Press; all rights reserved; doi: 10.1101/cshperspect.a022426

Cite this article as Cold Spring Harb Perspect Med 2015;5:a022426 
synchronous discharge of neurons in the brain. "Epileptic seizure" is used to distinguish a seizure caused by abnormal neuronal firing from a nonepileptic event, such as a psychogenic seizure. "Epilepsy" is the condition of recurrent, unprovoked seizures. Epilepsy has numerous causes, each reflecting underlying brain dysfunction (Shorvon et al. 2011). A seizure provoked by a reversible insult (e.g., fever, hypoglycemia) does not fall under the definition of epilepsy because it is a short-lived secondary condition, not a chronic state.

"Epilepsy syndrome" refers to a group of clinical characteristics that consistently occur together, with similar seizure type(s), age of onset, EEG findings, triggering factors, genetics, natural history, prognosis, and response to antiepileptic drugs (AEDs). The nonspecific term "seizure disorder" should be avoided.

Epilepsy is one of the most common neurologic conditions, with an incidence of approximately 50 new cases per year per 100,000 population (Hauser and Hersdorffer 1990). About $1 \%$ of the population suffers from epilepsy, and about one-third of patients have refractory epilepsy (i.e., seizures not controlled by two or more appropriately chosen antiepileptic medications or other therapies). Approximately 75\% of epilepsy begins during childhood, reflecting the heightened susceptibility of the developing brain to seizures.

\section{CLASSIFICATION OF SEIZURES AND EPILEPSIES}

The most recent International League Against Epilepsy (ILAE) classification of epileptic seizures and epilepsies (epilepsy syndromes), published in 2010, revises past classifications using terminology and concepts appropriate for the modern era (Berg et al. 2010; Berg and Millichap 2013; Muro and Connolly 2014). Seizures are divided into three categories: generalized, focal (formerly called partial), and epileptic spasms. Focal seizures originate in neuronal networks limited to part of one cerebral hemisphere. Generalized seizures begin in bilateral distributed neuronal networks. A seizure can begin focally and later generalize. Seizures can originate in the cortex or in subcortical structures. Using a detailed history, EEG findings, and ancillary information, a physician can often categorize the seizure/epilepsy type, after which an appropriate diagnostic evaluation and treatment plan is formulated.

The main subtypes of generalized seizures are absence, generalized tonic-clonic (GTC), myoclonic, and atonic (Table 1). Absence seizures (formerly called petit mal) involve staring with unresponsiveness to external verbal stimuli, sometimes with eye blinking or head nodding. GTC seizures (formerly called grand mal) consist of bilateral symmetric convulsive movements (stiffening followed by jerking) of all limbs with impairment of consciousness. Myoclonic seizures consist of sudden, brief ("lightning-fast") movements that are not associated with any obvious disturbance of consciousness. These brief involuntary muscle contractions may affect one or several muscles; therefore, myoclonic seizures can be generalized or focal. Atonic seizures involve the loss of body tone, often resulting in a head drop or fall.

The clinical manifestations of a focal seizure depend on the area of cortex involved. For example, a focal seizure arising from the occipital lobe may present with visual phenomena; from the precentral gyrus, with rhythmic clonic or tonic motor activity; and from the postcentral gyrus, with sensory symptoms, such as paresthesias. When consciousness is impaired during a focal seizure, that is, the patient is unable to respond normally to verbal or tactile stimuli, the seizure is classified as dyscognitive (formerly called complex partial); seizures arising from the temporal lobe are often dyscognitive. Some

Table 1. Epileptic seizures

Generalized seizures
Tonic-clonic
Absence
Typical
Atypical
Myoclonic
Atonic
Focal seizures
Epileptic spasms


seizures are preceded by an aura, which is a focal seizure wherein a patient retains awareness and describes motor, sensory, autonomic, or psychic symptoms. An aura precedes a focal dyscognitive or generalized seizure by seconds or minutes and is most often experienced by patients with temporal lobe epilepsy.

The origin of the third category of seizure type, epileptic spasms, is uncertain. Epileptic spasms are manifest by sudden extension or flexion of extremities, held for several seconds, and then recur in clusters. Epileptic spasms can occur at any age; when they begin in the first year of life, they comprise a syndrome called infantile spasms (IS) (West syndrome [WS]; see below).

Epilepsies (epilepsy syndromes) (Table 2) were previously classified according to their onset site (generalized or related to a specific cortical localization) and etiology, that is, whether the cause was known (symptomatic) or not known (idiopathic). Here, we use the 2010 revised guideline for classification of seizures and epilepsy (Berg et al. 2010). The updated system takes into account expanding knowledge of structural and genetic causes, and includes the ictal semiology (seizure type), syndrome diagnosis (if present), and degree of functional impairment. New classification schemes will continue to evolve as knowledge about epilepsy pathophysiology, and genetics emerges.

Table 2. Examples of epilepsy syndromes according to age of onset

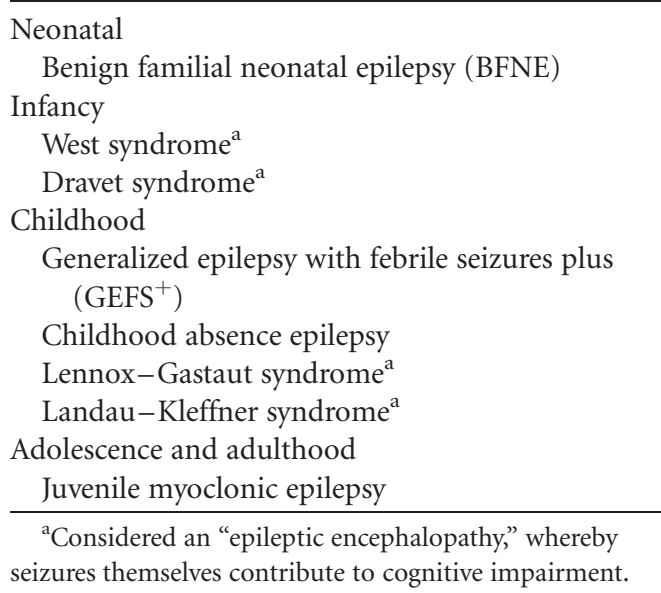

\section{PATHOPHYSIOLOGY AND GENETICS}

A seizure can be conceptualized as occurring when there is distortion of the normal balance between excitation (E) and inhibition (I) in the brain (Stafstrom 2010). This E/I imbalance can result from an alteration at many levels of brain function, from genes and subcellular signaling cascades to widespread neuronal circuits. The factors that alter E/I balance can be genetic or acquired. Genetic pathologies leading to epilepsy can occur anywhere from the circuit level (e.g., abnormal synaptic connectivity in cortical dysplasia) to the receptor level (e.g., abnormal $\gamma$-aminobutyric acid [GABA] receptor subunits in Angelman syndrome) to abnormal ionic channel function (e.g., potassium channel mutations in benign familial neonatal epilepsy [BFNE]). Similarly, acquired cerebral insults can alter circuit function (e.g., structural alteration of hippocampal circuitry following prolonged febrile seizures or head trauma). The developing brain is particularly prone to seizures for a variety of physiological reasons (see Berkovic 2015). Even in the normal developing brain, excitatory synaptic function develops before inhibitory synaptic function, favoring enhanced excitation and seizure generation. In addition, early in life, the neurotransmitter GABA causes excitation rather than inhibition (BenAri 2002; Pitkänen et al. 2015). These observations partly explain why the very young brain is especially susceptible to seizures. However, seizures cause less structural damage in the developing brain than in the adult brain (Holmes and Ben-Ari 1998).

There has been a recent explosion of new information about the genetic basis of epilepsy syndromes. Both monogenic and polygenic mutations can lead to epilepsy (Poduri and Lowenstein 2011). Many epilepsies have a complex genetic basis with multiple gene defects contributing to a state of altered cellular excitability, which underlies epilepsy. For example, copy number variants, which are de novo or inherited deletions or duplications $>1 \mathrm{~kb}$, are increasingly recognized as a source of genetic mutations in patients with epilepsy (Mullen et al. 2013; Olson et al. 2014). As genetics knowl- 
edge expands, there is hope that syndrome-specific therapeutic interventions can be designed (Thomas and Berkovic 2014).

\section{DIAGNOSTIC EVALUATION}

\section{History and Examination}

The history and neurologic examination are the cornerstones of the diagnosis of seizures and epilepsy, whereas laboratory evaluations serve as adjunctive tests. Important historical features include the clinical context in which the seizure occurred, including premonitory signs, details of the seizure itself, such as phenomenology, responsiveness, focal features, and the postictal state. Further inquiry centers on whether an epilepsy syndrome is present, guides the nature and extent of the evaluation, and determines treatment and prognosis.

The neurological examination assesses focal signs that might implicate or localize cerebral pathology. For example, increased tone on one side of the body could indicate pathology in the contralateral hemisphere, such as a cortical dysplasia. The general physical examination is also important to determine whether the patient has an underlying condition. For example, abnormal skin markings could indicate a neurocutaneous disorder in which epilepsy is common, such as tuberous sclerosis or neurofibromatosis.

\section{EEG}

An EEG is a recording of the brain's electrical activity. It can detect abnormal electrical activity, such as focal spikes or waves (consistent with focal epilepsy), or diffuse bilateral spike waves (consistent with generalized epilepsy). A routine EEG will, preferably, include wakefulness, drowsiness, and sleep because the prevalence of epileptiform abnormalities varies in these different states of consciousness. Hyperventilation and photic stimulation are activation procedures performed during an EEG to increase the yield of epileptic activity. Having a patient hyperventilate for $3 \mathrm{~min}$ has a high yield of leading to an absence seizure, related to the seizure-provoking effect of alkalosis (Schuchmann et al. 2006).
Photic stimulation may elicit paroxysmal epileptiform activity or even a generalized seizure in a person susceptible to generalized epilepsy (Verrotti et al. 2012). Simultaneous video-EEG monitoring for hours to days can increase the diagnostic yield or differentiate an epileptic seizure from a nonepileptic event. The EEG can be repeatedly normal in someone with epilepsy, especially if seizures begin in the frontal or temporal lobe. In such cases, intracranial EEG monitoring, usually in the context of presurgical evaluation, may be necessary to define a seizure focus. The diagnosis of epilepsy is based on clinical information and the EEG should be regarded as confirmatory, not diagnostic. The standard teaching is "treat the patient, not the EEG." An exception to this guideline is absence epilepsy in which brief generalized bursts of spike-wave activity, even if not associated with obvious clinical changes, imply a high likelihood of absence seizure recurrences that can go unrecognized.

\section{Neuroimaging}

Computed tomography (CT) and magnetic resonance imaging (MRI) scans are important adjuncts to the clinical examination and EEG in the evaluation of a person with seizures. Neuroimaging techniques are especially sensitive for central nervous system (CNS) structural lesions. Focal neurologic findings on examination (e.g., unilateral weakness, asymmetric reflexes) mandate neuroimaging.

MRI is more likely to show an abnormality in a patient with focal seizures, abnormal neurologic findings, or focal discharges on EEG. MRI is more sensitive than CT and is therefore preferred, especially for the detection of cortical malformation, dysgenesis, or hippocampal sclerosis. Quantitative, computer-assisted volume analysis of the temporal lobes may detect asymmetries that are not readily apparent on visual analysis of the scan. CT is valuable in the acute setting to detect hemorrhage, calcification, or tumors.

Several new imaging techniques are available to aid in the assessment of epilepsy (Kim et al. 2010). MRI abnormalities can be correlated directly with EEG activity. Functional MRI ( fMRI) 
takes advantage of blood oxygen level dependence (BOLD) to image neuronal activation and map interictal or ictal epileptiform activity and localize language and memory. Magnetic resonance (MR) spectroscopy measures the concentrations of a variety of neurochemicals in different brain regions and can sometimes assist in localizing a seizure focus. Positron emission tomography (PET) images the brain's regional use of glucose with asymmetries suggesting areas of interictal or ictal abnormality. Single-photon emission-computed tomography (SPECT) compares local blood flow discrepancies, information that is most useful when recorded during a seizure. Magnetoencephalography (MEG) assesses the brain's dynamic electromagnetic fields and can better localize epileptic dipoles, including those tangential to the scalp, which can be missed by conventional EEG (Caruso et al. 2013). These advanced modalities are used mainly in epilepsy centers for presurgical evaluations (Kay and Szaflarski 2014).

\section{Metabolic Evaluation}

The type of seizure and syndrome dictates the extent of the metabolic workup (Pearl 2009). For example, a child with IS or Lennox-Gastaut syndrome is more likely to have a metabolic or degenerative disorder than one presenting with simple partial seizures. In metabolic disorders, seizures are typically accompanied by other abnormalities, such as developmental delay, unexplained vomiting, or coma. In neonatal seizures, a metabolic evaluation is mandatory, including a screen of serum amino acids and urine organic acids, and blood lactate to screen for mitochondrial disease. In addition to its more common use to evaluate CNS infection, cerebrospinal fluid can be analyzed for glucose transporter defects (GLUT1 deficiency syndrome) (Pearson et al. 2013) and rare (but sometimes treatable) neurotransmitter defects (Pearl et al. 2007).

\section{Genetic Testing}

As the genetic basis of epilepsies becomes progressively unraveled, clinical testing will occupy an increasingly pivotal role in the clinic (Miche- lucci et al. 2012; Olson and Poduri 2014). At this point, genetic testing is available for several single genes, as well as complex genetic disorders (see Coulter and Steinhäuser 2015; Vezzani et al. 2015). A basic karyotype can be performed to evaluate for a chromosomal anomaly, especially in a patient with dysmorphic features. If a specific syndrome is suspected, an epilepsy panel of selected genes can be ordered (e.g., SCN1A for Dravet syndrome [DS]). Comparative genomic hybridization (CGH) microarray evaluates targeted chromosomal regions for copy number variants. When a genetic diagnosis is highly suspected, but other work up is unrevealing, the clinician can consider whole exome sequencing of the patient and parents, a technique with rapidly expanding clinical use, especially in epileptic encephalopathies of unknown etiology (Olson et al. 2014).

\section{SELECTED EPILEPSY SYNDROMES}

The most recent ILAE epilepsy classification dispenses with the dichotomies used in the 1989 classification (generalized vs. localization related, idiopathic vs. symptomatic) in favor of an organization according to pathogenesis (genetic vs. structural/metabolic/autoimmune) and age of onset (Berg et al. 2010). Examples of epilepsy syndromes are now provided. Details of epilepsy syndromes not described here can be found elsewhere (Engel and Pedley 2008; Nabbout and Dulac 2008).

\section{Electroclinical Syndromes with Age-Related Onset}

\section{BFNE}

BFNE is a neonatal epilepsy syndrome in which seizures begin in the first week of life. Seizures are focal clonic or focal tonic, often accompanied by apnea. They usually stop after a few days or weeks. Except for seizures, the infants are normal and evaluation fails to detect an etiology. The key to the diagnosis is a family history of newborn or infantile seizures that resolved. The prognosis of BFNE is good, although $\sim 10 \%-15 \%$ of affected infants continue to 
have seizures beyond the neonatal period, even into adulthood (Steinlein et al. 2007).

BFNE is the first epilepsy syndrome to be explained by a mutation in a voltage-gated ion channel gene. BFNE has been linked to two genes: KCNQ2 on chromosome $20 \mathrm{q}$ and KCNQ3 on chromosome 8q. These genes code for voltage-gated potassium channel subunits, which regulate the M-current, a muscarine-activated neuronal current that turns off potassium channels (Rogawski and Bazil 2008). The M-current stabilizes resting membrane potential; its dysfunction leads to increased neuronal excitability and seizures. It is not known why seizures in BFNE affect neonates and then resolve because the genetic defect is present throughout life.

\section{WS}

WS is characterized by the triad of epileptic spasms (usually during infancy, when it is called IS), an interictal EEG pattern called hypsarrhythmia, and intellectual disability. WS is an age-specific disorder, beginning primarily in the first year of life; the peak age of onset is between 4 and 6 mo. The duration of an epileptic spasm is intermediate between a myoclonic jerk (which is briefer) and a tonic seizure (which is more sustained). Spasms often occur in clusters of head nods, forceful flexion, or extension of the trunk and limbs. They frequently occur during sleep transitions, especially on awakening.

The interictal EEG pattern in WS is called hypsarrhythmia, a disorganized, "chaotic" pattern of very high voltage slow waves and spikes over multiple cortical areas. The classic ictal EEG pattern is a generalized slow wave followed by background voltage attenuation in all channels ("electrodecremental response"), accompanied by a clinical spasm.

Most cases of WS have an identifiable cause, such as hypoxia-ischemia, intracranial hemorrhage, CNS infection, developmental brain anomaly, or inborn metabolic error. Tuberous sclerosis complex (TSC) has an especially high incidence of IS (up to $50 \%$ of TSC patients).

Adrenocorticotrophic hormone (ACTH) and corticosteroids are the primary drugs used to treat IS. The anticonvulsant mechanism of ACTH is not known; it may work via the hypothalamic-pituitary axis or directly affect neuronal membrane excitability (Stafstrom et al. 2011). Vigabatrin, a GABA transaminase inhibitor, is highly effective for spasms in children with TSC. Infants with focal-onset spasms, such as those caused by cortical dysplasia, may benefit from resective surgery.

WS is an epileptic encephalopathy with a poor prognosis. At least two-thirds of affected children have intellectual disability. With age, the seizures often change from spasms to other seizure types, such as those seen in LennoxGastaut syndrome (see below). Several animal models of IS have been reported recently, raising hope that elucidating the pathophysiology of IS will lead to more efficacious treatments (Stafstrom 2009; Swann and Moshe 2012; Lado et al. 2013).

\section{Febrile Seizures Plus}

Children with febrile seizures plus $\left(\mathrm{FS}^{+}\right)$(formerly called generalized epilepsy with febrile seizures plus $\left[\mathrm{GEFS}^{+}\right]$) have febrile seizures beyond the age at which febrile seizures usually stop ( $\sim 5 \mathrm{yr})$. In addition, these children may develop additional afebrile seizure types, including GTC, absence, and myoclonic. Therefore, this syndrome differs from ordinary febrile seizures (see below) and represents a genetic predisposition to epilepsy. In $\mathrm{FS}^{+}$, the outcome is variable; seizures resolve in some children, but persist in others. In different families, genetic defects have been identified in neuronal sodium channels (Escayg et al. 2000) and GABA receptors (Macdonald et al. 2010). Many patients with $\mathrm{FS}^{+}$have mutations in the $\alpha 1$ subunit of the voltage-gated sodium channel gene, SCN1A (Steinlein 2014).

\section{DS}

DS, previously called severe myoclonic epilepsy of infancy, is a rare epilepsy syndrome in which children present with seizures before 18 mo of age (Dravet et al. 2005). The initial seizure often occurs with a fever and has a hemiclonic semi- 
ology. Later, other seizure types occur and the child shows developmental regression. Seizures tend to be refractory to medications, although stiripentol has shown some efficacy; sodium channel blockers must be avoided.

About $70 \%-80 \%$ of patients with DS have a mutation in the SCN1A gene, mostly sporadic, with haploinsufficiency causing nonfunctional sodium channels. Therefore, the spectrum of SCN1A mutations in epilepsy spans from mild $\left(\mathrm{FS}^{+}\right.$- missense mutations) to severe (DS-truncating mutations) (Escayg and Goldin 2010). The presence of SCN1A mutations in multiple epilepsy syndromes has generated considerable research interest. Mice with knockout of SCN1A replicate many clinical features of DS (Oakley et al. 2011). The cellular defect may be abnormal sodium channels in cortical interneurons, allowing increased firing of downstream excitatory pyramidal neurons, which are released from inhibitory control (Yu et al. 2006). Several laboratories are pursuing potential methods to remediate the effect of the SCN1A mutation (Liu et al. 2013; Lenck-Santini and Scott 2015).

\section{Lennox-Gastaut Syndrome}

Lennox-Gastaut syndrome (LGS) begins between the ages of 1 and 6 yr. Patients develop medically intractable seizures (up to hundreds per day), constituting an epileptic encephalopathy. LGS characteristics include: (1) slow spikewave EEG pattern $(1.5-2.5 \mathrm{~Hz}),(2)$ intellectual disability, and (3) multiple seizure types (e.g., tonic, GTC, atypical absence, atonic, tonic, myoclonic).

Tonic seizures consist of periods of sustained muscle contractions and are especially frequent during sleep. Atonic (astatic) seizures, or drop attacks, occur without warning and often result in head or face injuries. Atypical absences occur frequently in children with LGS. These have a gradual onset and cessation during which the child appears confused with behavioral arrest. It can be difficult to tell when one seizure ends and the next one begins because alertness and activity level may not improve between epileptiform bursts, which occur in long runs during wakefulness and even more frequently during sleep.

Children with LGS are already handicapped neurologically. The numerous LGS etiologies overlap those of WS and include hypoxic brain injury, cerebral dysgenesis, and neurocutaneous disorders. The encephalopathy in the majority of children with LGS is static, although a degenerative disorder, such as neuronal ceroid lipofuscinosis, can present as LGS.

Seizures in LGS patients are notoriously refractory to AEDs. Drug therapy is individualized to seizure type and frequency (Hancock and Cross 2013). Patients may benefit from valproate, clonazepam, lamotrigine, topiramate, rufinamide, lacosamide, clobazam, or felbamate. Because of the intractability of the seizures, there is a tendency to place patients on multiple AEDs. This polypharmaceutical approach often causes drug toxicity with somnolence, fatigue, nausea, ataxia, and rarely results in optimal seizure control.

Children with LGS have a poor neurologic prognosis. Over time, the atonic, myoclonic, and atypical absence seizures may decrease, but GTC seizures increase and partial seizures emerge. In addition to debilitating seizures, intellectual impairment hinders children with LGS from leading independent lives. The lack of an experimental model hinders progress in this disorder. One potentially informative animal model mimics atypical absence seizures (Cortez et al. 2001).

\section{Landau-Kleffner Syndrome}

Landau-Kleffner syndrome (LKS) (acquired epileptic aphasia) is a rare epilepsy in which a child loses previously acquired language abilities because of seizures or epileptiform abnormalities on EEG. In its pure form, LKS occurs in previously normal children with normal language development who gradually lose the ability to understand spoken language and produce speech (Landau and Kleffner 1957). More recently, the syndrome has expanded to include behavioral and cognitive deterioration, including autistic symptoms. Regression of social and language skills is frequently seen in children 
with autism, with or without accompanying seizures, so the differentiation of autism and LKS can be difficult. In LKS, compared with autism, social skills are better preserved. The pathophysiology of LKS is unknown. Imaging studies are generally negative although PET studies have shown bitemporal abnormalities, supporting the hypothesis that language-related brain regions are dysfunctional in LKS (Issa 2014).

EEG abnormalities in LKS may include generalized, focal, or multifocal spikes or spike waves. If focal, discharges commonly involve one or both temporal or perisylvian regions. One hypothesis is that the epileptiform discharges interfere with language production; alternatively, both the language dysfunction and EEG abnormalities might be independent consequences of the same underlying brain pathology. Successful treatment of the seizures or EEG discharges is not usually accompanied by language or behavioral improvement. The outcome is variable; some children recover completely, usually in adolescence, whereas others have persistent aphasia in adulthood. The seizures usually respond readily to AEDs (e.g., valproate, benzodiazepines), although the language impairment does not (Van Bogaert 2013). Treatment with steroids or subpial resection is controversial.

\section{Childhood Absence Epilepsy (CAE)}

Absence seizures, characterized by staring and diminished responsiveness, can be part of several epilepsy syndromes, including CAE and juvenile myoclonic epilepsy (JME). Note that "absence" refers to both a seizure type and an epilepsy syndrome. CAE onset is between 4 and $10 \mathrm{yr}$ of age. The seizures start abruptly and, generally, last from 5 to $20 \mathrm{sec}$. When a seizure ends, the patient immediately resumes prior conversation or activity. Because absence seizures are brief and nonconvulsive, they can be easily missed or misdiagnosed.

The frequency of absence seizures varies from a few to hundreds per day. Stress and fatigue increase their frequency. Most children with typical absence seizures have a normal neurologic examination and intelligence, although school performance may be impaired if seizures are frequent.

The EEG background is normal, whereas the seizure itself is accompanied by generalized $3-\mathrm{Hz}$ spike-wave complexes. This EEG abnormality is a marker for genetic susceptibility to absence epilepsy. Hyperventilation is a potent activator of absence seizures, and this simple test is used in the clinic to diagnose absence seizures and assess treatment effectiveness.

The pathophysiology of absence seizures involves altered function of thalamocortical circuits, with thalamic relay neurons firing abnormally owing to calcium channel dysfunction (Cain and Snutch 2013). Ethosuximide and valproic acid (VPA) are effective for treating absence seizures (Glauser-Menachem et al. 2013). Both drugs block low-threshold calcium currents in thalamic neurons (Coulter et al. 1989). CAE (and other genetic generalized epilepsies) has a complex genetic basis with only a few percent transmitted monogenically. The prognosis of CAE is good with $\sim 75 \%$ of children outgrowing the absence seizures during adolescence.

\section{JME}

JME is an epilepsy syndrome that typically begins in adolescence and consists of myoclonic or GTC seizures in an otherwise normal individual. The myoclonic jerks may cause the patient to drop or fling objects, especially in the morning. GTC seizures occur in as many as $90 \%$ of patients with JME, and the syndrome often presents with these. The myoclonic and GTC seizures often occur soon after awakening. Up to $35 \%$ of patients with JME also have absence seizures. Seizures are exacerbated by fatigue, sleep deprivation, and alcohol use.

The neurologic examination and intelligence are usually normal in JME. Multifactorial inheritance is presumed. Some studies have linked JME to chromosome 6p, a locus that appears to be dominantly inherited, but a responsible gene has not yet been identified, and this mutation accounts only for a small fraction of patients (Michelucci et al. 2012).

The interictal EEG in JME shows characteristic bursts of fast (3.5- to 6-Hz) spike-wave 
complexes. Photic stimulation may activate these epileptiform discharges. Valproate is the most effective AED, but, in females, other broad-spectrum AEDs are preferable (levetiracetam, lamotrigine). Long-term treatment is usually required.

\section{Epilepsy Syndromes Caused by Structural/ Metabolic/Autoimmune Causes}

Epilepsy syndromes, previously called "symptomatic localization-related," are those in which seizures arise in a focal brain region caused by an acquired or congenital lesion. Etiologies include tumor, scar (e.g., hippocampal sclerosis), cortical dysplasia, porencephalic cyst, and vascular malformation. The seizure semiology is related to the region of brain affected; seizures often begin focally and then generalize. The interictal EEG will show focal spikes, sharp waves, or slowing, related to the area of brain involved. If neuroimaging results, EEG evidence of seizure onset, and ancillary data (e.g., neuropsychological findings) align, surgical intervention is considered.

\section{Temporal Lobe Epilepsy}

The syndrome of mesial temporal sclerosis is a pertinent example of a structural lesion (hippocampal scarring), in which seizures often become intractable and for which surgery is a viable option (Thom et al. 2010; Bernhardt et al. 2013). Seizures originate in the medial temporal region with such manifestations as posturing, altered responsiveness, and memory/behavior change. Spread of seizure discharges beyond the hippocampus is common. Seizures often become intractable and affective comorbidities are frequent. When two medications fail, a surgical evaluation should be undertaken. Extensive laboratory investigation has been performed to understand the mechanisms of seizure genesis and spread. Impaired GABAergic inhibition, enhanced synaptic excitation via axonal sprouting, and changes in ion channel distribution and function have all been implicated in the pathophysiology of temporal lobe epilepsy, and genetic factors may also play a role (Liu et al. 1995;
Buckmaster 2004; Dudek and Sutula 2007; Joshi et al. 2013).

\section{Childhood Hemispheric Epilepsy Syndromes}

Some important childhood epilepsy syndromes involve an entire hemisphere. Rasmussen's encephalitis is a focal encephalitis that affects only one hemisphere and results in progressive hemiparesis, intractable epilepsy (focal seizures that can progress to become continuous, called epilepsia partialis continua), and cognitive decline (Varadkar et al. 2014). Rasmussen's encephalitis might have an autoimmune basis, but the exact etiology has not been defined. The unilateral pathology may be a result of focal breakdown of the blood-brain barrier. Neuroimaging shows progressive unilateral cortical atrophy. Another hemispheric syndrome, SturgeWeber syndrome (SWS; encephalotrigeminal angiomatosis), consists of a hemispheric vascular malformation, leading to intractable epilepsy and hemiparesis. Mutations in GNAQ, a gene governing angiogenesis, have recently been identified in SWS (Shirley et al. 2013). Some authorities feel that early surgery (hemispherectomy) affords a better prognosis in hemispheric epilepsy syndromes (Hartman and Cross 2014).

\section{Metabolic, Mitochondrial, and Autoimmune Epilepsies}

Epilepsies caused by a metabolic, mitochondrial, or autoimmune etiology are increasingly recognized. Any alteration of neuronal energy metabolism or use could result in E/I imbalance and seizures. The role of autoantibodies to a variety of cellular proteins in patients with heretofore undiagnosed neurological deterioration is shedding new light on the ways in which epilepsy can manifest (Davis and Dalmau 2013; Miya et al. 2014; Holmes 2015).

\section{Other}

\section{Neonatal Seizures}

Neonatal seizures, occurring in the first $30 \mathrm{~d}$ of life or before $44 \mathrm{wk}$ postconception in prema- 
ture babies, represent a special class because of their age-specific characteristics, wide range of etiologies, and unique pathophysiology. Seizures may be the first and only sign of CNS dysfunction in a newborn, so their recognition is critical.

Four types of neonatal seizure semiology are described based on behavioral observations: subtle, generalized tonic, focal or multifocal clonic, and myoclonic. Subtle seizures may include repetitive oral-buccal-lingual movements, such as sucking, pedaling movements of the legs or arms, or eye deviation. Subtle seizures are often associated with severe CNS insults. Neonatal tonic seizures involve posturing with intermittent tonic extension of the arms and legs; they are usually associated with severe brain lesions and most often occur in preterm infants. Clonic seizures consist of rhythmic jerking of groups of muscles in a focal or multifocal pattern. In multifocal clonic seizures, movements migrate from one part of the body to another. Focal seizures may be seen with localized brain malformations or insults, such as a perinatal stroke, as well as in disorders affecting the brain diffusely, such as asphyxia, metabolic derangement, or infection. As a result of immature myelination and cortical organization, the neonatal brain is unable to sustain generalized epileptiform discharges, so GTC and absence seizures do not occur.

Simultaneous video-EEG monitoring can help to differentiate behaviors with EEG correlates ("epileptic seizures") from behaviors that do not have associated EEG changes. Focal clonic seizures have the highest correlation with EEG ictal abnormalities. Many behaviors considered to be subtle seizures on clinical grounds (e.g., chewing or pedaling movements) have no associated EEG abnormalities, suggesting that these behaviors are not epileptic in nature. Subtle or tonic seizures may represent brainstem dysfunction or epileptic seizures originating from deep subcortical structures not recordable on surface EEG.

The neonatal EEG is usually not specific for a particular etiology, but it may supply clues about the severity and time course of a CNS insult. Epileptic discharges often occur without overt clinical manifestations ("uncoupling" of electrographic and clinical seizures). For prognostic purposes, EEG background patterns and sleep - wake cycles are especially important. Amplitude-integrated EEG (aEEG) is a new technique allowing continuous bedside sampling of a limited number of EEG channels; aEEG is proving to be quite reliable in documenting probable seizure events (Glass et al. 2013).

Establishing the etiology of a neonatal seizure is critical because the cause determines the therapy and is highly correlated with outcome. Major causes of neonatal seizures include hypoxic-ischemia (H-I), hypocalcemia, hypoglycemia, hyponatremia, intracranial hemorrhage, infection, congenital malformations, genetic factors, inherited metabolic disorders, and drug withdrawal. H-I, mostly occurring before delivery, is the most common cause of neonatal seizures.

The decision to treat an infant with recurrent seizures is based on the seizure duration and frequency, associated autonomic dysfunction, etiology, and EEG abnormalities. If seizures are brief and not associated with autonomic instability, treatment may be deferred or the infant treated with a short-acting benzodiazepine. Conversely, neonates with frequent seizures, especially if they interfere with ventilation, require prompt and vigorous treatment.

Phenobarbital has been the primary drug used to treat neonatal seizures, but it is effective $<50 \%$ of the time (Painter et al. 1999). Phenobarbital or phenytoin sometimes suppresses clinical seizures, but electrographic seizures continue ("uncoupling"). Any drug that targets GABA receptors (barbiturates, benzodiazepines) may be ineffective or even exacerbate seizures because of the depolarizing action of GABA in the neonatal brain (Staley 2006; Berkovic 2015; Pitkänen et al. 2015). Active research is attempting to untangle the role of potassium-chloride cotransporters and their developmental profile in the GABA depolarizing-to-hyperpolarizing switch; inhibitors of these cotransporters might have clinical utility (Löscher et al. 2013). Newer AEDs, such as levetiracetam, may be effective, but there is an urgent need for more effective neonatal seizure treatments. 


\section{Febrile Seizures}

Febrile seizures occur in children from $\sim 6$ mo to $5 \mathrm{yr}$ of age. Although the exact pathophysiology is unknown, febrile seizures represent an age-dependent response of the developing brain to fever. There is a genetic association, with febrile seizures occurring 2-3 times more frequently in affected families than in the general population. Overall, febrile seizures occur in $2 \%-5 \%$ of children in the susceptible age range.

The two main types of febrile seizures are: (1) simple, and (2) complex or complicated. Simple febrile seizures are brief $(<15 \mathrm{~min})$, generalized, and do not recur within $24 \mathrm{~h}$ of the first one. Complicated febrile seizures are either prolonged ( $>15 \mathrm{~min}$ ), have focal components (e.g., begins on one side of the body or involves lateralized eye deviation), or recur within $24 \mathrm{~h}$. Simple febrile seizures do not require treatment, nor do most complicated febrile seizures.

A child who has had a single simple febrile seizure has a $33 \%$ chance of another febrile seizure with a subsequent fever. If a child has had two simple febrile seizures, there is a $50 \%$ chance of a third febrile seizure. The recurrence risk for additional febrile seizures is greatest if a child has had a first febrile seizure $<12$ mo of age or there is a family history of febrile seizures.

The more concerning risk is for the development of afebrile seizures (epilepsy). A significant proportion of adults with temporal lobe epilepsy (caused by mesial temporal sclerosis) had a prolonged febrile seizure as a child (Patterson et al. 2014). After a simple febrile seizure, the risk for epilepsy later in life is only slightly higher than for the general population, $\sim 2 \%$. The risk of developing epilepsy after febrile seizures varies up to $\sim 9 \%$ and is greatest if the child has preexisting neurologic impairment, such as developmental delay or cerebral palsy, a family history of epilepsy, or a complicated febrile seizure (prolonged, focal, recurrent) (Pavlidou and Panteliadis 2013).

An ongoing multicenter study (FEBSTAT) is investigating the consequences of febrile status epilepticus in a large cohort using longitudinal clinical and MRI data (Lewis et al. 2014). A complete understanding of febrile seizures will also require animal models to gain greater insight into the effects of hyperthermia and feverrelated immunological changes in the developing brain (McClelland et al. 2011).

\section{NONEPILEPTIC DISORDERS THAT MIMIC SEIZURES}

Numerous paroxysmal behaviors mimic epileptic seizures by history or clinical presentation (Obeid and Mikati 2007). A few of the more common disorders are mentioned here. It is important to distinguish epileptic from nonepileptic behaviors because some nonepileptic phenomena respond to medications other than AEDs and others require no specific treatment other than reassurance or avoidance of the circumstances that precipitate the spell.

\section{Nonepileptic Seizures (NES)}

NES, also called psychogenic seizures or pseudoseizures, are paroxysmal changes in motor activity or behavior that resemble epileptic seizures, but have no EEG correlate. Although they are not epileptic seizures, NES can be disabling and often reflect major underlying psychopathology (Lortie 2013).

NES present with a variety of clinical forms. Many resemble GTC seizures, but the two sides of the body are more likely to jerk out of phase with each other. GTC activity in the setting of preserved consciousness favors a nonepileptic event. However, caution is warranted as some behaviors previously thought to be NES are actually epileptic events. For example, seizures originating in the supplementary area (SMA) of the frontal lobe involve bilateral motor activity with preserved consciousness. Compared with NES, SMA seizures are briefer, more stereotyped, and often occur during sleep. Seizures originating in the orbitofrontal region are now recognized to include screaming, affective changes, such as intense fear, bilateral nonrhythmic limb movements, and even sexual automatisms, for example, pelvic thrusting. Such behaviors were previously considered to reflect NES. These observations underscore the difficulty of differentiating between epileptic sei- 
zures and NES on clinical grounds. Video-EEG monitoring is helpful to separate the two entities. Of note, NES and epileptic seizures may coexist in the same patient.

The patient and family are assured that the symptom (seizure) is "real," but does not involve abnormal neuronal discharges, so therapy is designed to address the underlying psychological issues. The main therapeutic goal is to teach the patient alternative coping skills, so that anxiety or psychological stress does not manifest in such a maladaptive fashion (LaFrance et al. 2013). The pathophysiology of NES is uncertain, and an important research question is how and why psychological stress results in seizure-like behaviors.

\section{Breath-Holding Spells (BHS)}

Despite their name, BHS are involuntary reflex responses. BHS are maximal in preschoolers and are, typically, outgrown by school age. Two types of BHS are cyanotic (also called cyanotic infantile syncope) and pallid (also called pallid infantile syncope or reflex anoxic seizures).

Cyanotic BHS, the more common type, are precipitated by anger or frustration. The hallmark is crying, during which the child will stop breathing (in expiration), become cyanotic, and lose consciousness. At that point, the child may become rigid, limp, or even shake, raising concern about a seizure. The pathogenesis of cyanotic BHS is complex, probably involving an interaction between hyperventilation, Valsalva maneuver, expiratory apnea, and intrinsic pulmonary mechanics.

Pallid BHS are more likely to be provoked by fright or an unpleasant stimulus (such as mild trauma). A gasp is followed by loss of consciousness, pallor, bradycardia, diaphoresis, and limpness. Pallid BHS result from vagus nerve-mediated cardiac inhibition, causing diminished cerebral blood flow.

Neither type of BHS is associated with an increased predisposition to epilepsy, although seizure activity can occur at the end of a BHS. These "seizures" manifest as tonic stiffening of the extremities, sometimes with brief clonic jerking, and are not epileptic. Instead, they represent the brain's response to acute hypoxia. They terminate spontaneously and do not require anticonvulsant treatment. Evaluation with an EEG is usually not needed. An electrocardiogram is obtained to rule out prolonged QT syndrome. Management of BHS consists mainly of reassurance that the spells will be outgrown.

\section{Syncope}

Syncope (fainting) can usually be distinguished from an epileptic seizure by history. Attacks may be preceded by warning ( presyncopal) signs, such as lightheadedness, blurred vision, pallor, nausea, or diaphoresis. These warning signs are followed by a loss of consciousness and slow slump to the ground, as opposed to a more abrupt fall seen with a myoclonic or atonic seizure. Late in a syncopal spell, there may be a brief tonic or clonic seizure secondary to cerebral hypoperfusion and hypoxia; these are not epileptic seizures. Consciousness is regained rapidly, compared with a more prolonged postictal state after an epileptic seizure. The seizure that follows BHS or syncope engages neural circuitry that produces GTC activity, but the mechanisms underlying this hypoxia-related seizure activity warrant further clarification.

Syncope is caused by transient reduction of cerebral blood flow as a result of an irregular heart rate (an arrhythmia causing decreased cardiac output), decreased venous return (orthostasis or Valsalva), or vasovagal mechanism (fright, pain, emotional upset). Vasovagal attacks often occur in a hot environment. The EEG is usually normal. The key to treatment is the avoidance of precipitating factors.

\section{Parasomnias}

Parasomnias are sleep disorders that sometimes mimic seizures. Night terrors, a common parasomnia, occur in children from 18 mo to $8 \mathrm{yr}$ of age. In early (non-REM) sleep, the child awakens with inconsolable screaming, sweating, and nonrhythmic flailing of extremities, followed by return to sleep and no memory of the episode. 
There is often a family history of night terrors. The diagnosis is based on clinical history; video-EEG is rarely needed. The main differential diagnosis is nightmares (that occur during REM sleep) and nocturnal epileptic seizures of frontal lobe origin. The predisposition to epileptic seizures during sleep-state transitions, increased epileptiform activity in sleep, and risk of seizures with sleep deprivation all indicate an intimate relationship between sleep and epilepsy that is in need of further research.

\section{COMORBIDITIES}

Epilepsy is more than spontaneous recurrent seizures and should be considered a spectrum disorder. For many patients and families, the burden of the disease is largely caused by comorbid conditions, including behavioral and psychiatric disorders, such as depression, anxiety, learning disabilities, attention-deficit hyperactivity disorder, intellectual disability, and autism. These comorbidities, previously considered to be secondary to uncontrolled seizures or medication adverse effects, are now recognized as an integral part of the disorder, sometimes even preceding the seizures and attributable to an underlying disorder of neuronal networks (Brooks-Kayal et al. 2013). Even a single seizure can alter neurodevelopment by modifying receptor expression and distribution in the absence of neuronal death, leading to cognitive and behavioral changes (Cornejo et al. 2007). Understanding the pathophysiological link between these associated conditions and the epilepsies could have a major impact on the life of people living with epilepsy, and should be considered a research priority.

Depression is the most frequent psychiatric comorbidity and, interestingly, is associated with hippocampal and limbic dysfunction, structures commonly implicated in epileptic circuits. The association between epilepsy and depression is described as bidirectional: epilepsy patients with depression are more frequently refractory and people with epilepsy are more likely to develop depression. About $10 \%$ of adults with epilepsy have bipolar disorder and up to $30 \%$ have depression (Kanner 2013).
These symptoms are also associated with an increased suicide risk. This is also the case in children when symptoms are even less recognizable, but studies have shown that children with newly diagnosed epilepsy are almost three times more likely to have a mood disorder than controls. Anxiety is also difficult to diagnose clinically in patients with epilepsy because of the unpredictable nature of the disease, leading to some form of anxiety.

Nonpsychiatric comorbidities also affect this population. In the Centers for Disease Control and Prevention (CDC) National Health Interview Survey, adults with epilepsy had a higher prevalence of cardiovascular and respiratory disorders, diabetes, inflammation, obesity, and other disorders (e.g., headache, migraine, arthritis) (Strine et al. 2005). Persons with epilepsy are also at increased risk for early mortality and sudden unexplained death in epilepsy (SUDEP) (Surges and Sander 2014).

Recently, the impact of seizure medication on bone health has become a major concern. Patients with epilepsy are at high risk for fractures because of lower bone mineral density (BMD) (Beerhorst et al. 2013). Phenytoin, phenobarbital, and carbamazepine appear to be the antiseizure medications that lead to a reduction in BMD via induction of the CYP450 enzyme system results, but osteopenia has also been reported with non-enzyme-inducing AEDs.

\section{TREATMENT: GENERAL PRINCIPLES}

With an armamentarium of $>20$ drugs, up to $70 \%$ of newly diagnosed people living with epilepsy can be successfully treated. Drugs used to treat epilepsy work by decreasing the electrical activity of the brain, either by preventing neuronal depolarization by blocking sodium channels or calcium channels, enhancing potassium channel function, inhibiting excitation mediated by the neurotransmitter glutamate, or promoting inhibition mediated by GABA (Table 3 ) (see Bui et al. 2015). The efficacy of these medications varies based on etiology. Patients with no identified etiology are most likely to be controlled, especially if they have a normal developmental history and neurological examination. 
A neurologist's decision to begin one seizure medication over another is based on seizure type, age, other medical conditions, and potential side-effect profile. Often, a wide-spectrum medication is used as seizure description by a witness may be lacking. Levetiracetam has become very popular in recent years as first-line therapy because of its efficacy, easy titration, and well-recognized side-effect profile. Previously, carbamazepine was the first choice for focal seizures, whereas valproic acid was the first choice for generalized seizures.

Table 3. Mechanisms of action of selected AEDs

\begin{tabular}{|c|c|}
\hline Mechanism & Antiepileptic drugs \\
\hline $\begin{array}{l}\text { Block repetitive } \\
\text { activation of sodium } \\
\text { channels }\end{array}$ & $\begin{array}{l}\text { Phenytoin, } \\
\text { carbamazepine, } \\
\text { oxcarbazepine, } \\
\text { lamotrigine, } \\
\text { topiramate }\end{array}$ \\
\hline $\begin{array}{l}\text { Enhance slow } \\
\text { inactivation of sodium } \\
\text { channels }\end{array}$ & Lacosamide, rufinamide \\
\hline $\begin{array}{l}\text { Enhance activity of } \\
\gamma \text {-aminobutyric acid } \\
\left(\mathrm{GABA}_{\mathrm{A}}\right) \text { receptors }\end{array}$ & $\begin{array}{l}\text { Phenobarbital, } \\
\text { benzodiazepines, } \\
\text { clobazam }\end{array}$ \\
\hline $\begin{array}{l}\text { Block } N \text {-methyl-D- } \\
\text { aspartate (NMDA) } \\
\text { receptors }\end{array}$ & Felbamate \\
\hline $\begin{array}{l}\text { Block } \alpha \text {-amino-3- } \\
\text { hydroxy-5-methyl-4- } \\
\text { isoxazole propionic } \\
\text { acid (AMPA) } \\
\text { receptors }\end{array}$ & Perampanel, topiramate \\
\hline $\begin{array}{l}\text { Block T-calcium } \\
\text { channels }\end{array}$ & Ethosuximide, valproate \\
\hline $\begin{array}{l}\text { Block N- and L-calcium } \\
\text { channels }\end{array}$ & $\begin{array}{c}\text { Lamotrigine, topiramate, } \\
\text { zonisamide, valproate }\end{array}$ \\
\hline Modulate H-currents & Gabapentin, lamotrigine \\
\hline $\begin{array}{l}\text { Block unique binding } \\
\text { sites }\end{array}$ & $\begin{array}{l}\text { Gabapentin, } \\
\text { levetiracetam }\end{array}$ \\
\hline $\begin{array}{l}\text { Inhibit carbonic } \\
\text { anhydrase }\end{array}$ & Topiramate, zonisamide \\
\hline $\begin{array}{l}\text { Open potassium } \\
\text { channels (KCNQ } \\
[\mathrm{Kv} 7])\end{array}$ & Retigabine \\
\hline $\begin{array}{l}\text { Inhibit GABA } \\
\text { transaminase }\end{array}$ & Vigabatrin \\
\hline Inhibit GABA reuptake & Tiagabine \\
\hline
\end{tabular}

As a general principle, medication should be started at a low dose to avoid side effects. Dose increases can be performed at regular intervals if needed. The goal is to control seizures with the lowest dose. When a first drug fails, most clinicians will choose to add on a second drug, later deciding whether or not to withdraw the initial medication. A proper trial is considered to be 2 mo at a therapeutic, well-tolerated dose. Because of drug interactions, combination therapy has the potential of high toxicity; however, some combinations display particular efficacy, such as lamotrigine plus valproic acid for generalized seizures.

Because of their mechanism of action, all seizure medications have CNS side effects. For example, sleepiness is a common side effect of almost all AEDs. Lamotrigine is fairly well tolerated, but requires a very slow dose titration. Some physicians consider stopping a medication if seizures have not recurred over the previous 2 yr or more. If medication fails to control seizures, other options include dietary therapy (ketogenic diet), resective epilepsy surgery (lesionectomy, hemispherotomy), and palliative epilepsy surgery (stimulation therapy, callosotomy). The role of immune therapy for refractory epilepsy is still being defined.

Lifestyle adjustment is a crucial aspect of epilepsy management. Optimizing sleep, improving medication compliance, and reducing stress can significantly improve epilepsy outcome. Also important are prevention efforts to reduce causes of symptomatic epilepsies, such as head trauma, perinatal injury, and brain infections like neurocysticercosis.

Finally, health professionals need to advocate for people with epilepsy. Even today, stigma and discrimination represent significant barriers to a normal life for people living with epilepsy.

\section{PROGNOSIS}

Of persons with epilepsy, $60 \%-70 \%$ will see their seizures controlled by medication. In most cases, treatment is deferred until a second seizure occurs. The overall risk of recurrence following a single seizure varies between $27 \%$ and 
71\%. A meta-analysis found that the average risk of seizure recurrence was $40 \%$ in prospective studies versus $52 \%$ in retrospective studies (Berg and Shinnar 1991). Of these recurrences, $80 \%$ occur within the first $2 \mathrm{yr}$ of the initial seizure. Similar numbers are observed in children (Shinnar et al. 2000). As medical treatment has the potential for significant adverse events, the benefits for treatment are clearer after a second unprovoked seizure when the risk of recurrence within $1 \mathrm{yr}$ is doubled (Hauser et al. 1998; Shinnar et al. 2000). There is no evidence that early treatment affects long-term prognosis, but the outcome of children who have experienced more than 10 unprovoked seizures before treatment appears worse (Camfield et al. 1996).

About $70 \%$ of children will achieve a period of remission of at least $2 \mathrm{yr}$ without a seizure. At that time, one can consider stopping medication, especially if there is no identified underlying etiology and the child is developing well. Of those, $20 \%-25 \%$ will recur either when seizure medication is being withdrawn (50\%) or spontaneously (Sillanpää and Schmidt 2009). Of all patients, $37 \%$ will have easily controlled seizures from the onset, $25 \%$ will have refractory seizures from the onset, and $38 \%$ will have a remitting relapsing pattern. Patients with no identified etiology have a better outcome than those with a structural, metabolic, or genetic etiology.

An analysis of 14 AED withdrawal studies found that the recurrence rate following $\mathrm{AED}$ discontinuation ranged from $12 \%$ to $66 \%$ (mean 34\%), and reinstatement of treatment was successful in obtaining further remission in $\sim 80 \%$ with no significant differences between age groups. A second remission may, however, take many years to achieve, whereas, in $19 \%$, the reintroduction of medication did not control the seizures as well as before. Up to $23 \%$ of those discontinuing treatment go on to develop intractable epilepsy. Risk factors for subsequent poor treatment outcome are symptomatic focal epilepsy and cognitive deficits (Chadwick et al. 1996; Schmidt and Löscher $2005)$. Finally, $\sim 5 \%$ of those with refractory epilepsy will achieve a remission of 12 mo when medication is adjusted, although about one- half will subsequently relapse (Neligan et al 2012).

\section{CONCLUSIONS}

This review introduced the concepts of clinical epilepsy to allow neuroscientists to assess the state of the field and formulate relevant research questions. Many clinical conundrums in need of attention by researchers were discussed in a recent opinion paper in which the following topics were deemed ripe for neuroscientific investigation: the roles of genes versus acquired factors in seizure predisposition; how epilepsy develops in an otherwise normal brain and how to prevent the consequences of seizures, for example, following a brain injury (epileptogenesis, neuroprotection); methods to better localize seizure onset and identify at-risk circuits with a goal of surgical intervention; how to predict (and thereby avert) seizure occurrence; optimization of medication for specific ages and epilepsy syndromes; novel methods of drug delivery to vulnerable circuits; and interventions to relieve comorbidities during postictal and interictal periods, which comprise the majority of a patient's daily function, but disproportionately affect the quality of life (Fisher 2009). Considerable progress has been made, but there is obviously much work remaining.

\section{REFERENCES}

* Reference is also in this collection.

Beerhorst K, van der Kruijs SJ, Verschuure P, Tan IY, Aldenkamp AP. 2013. Bone disease during chronic antiepileptic drug therapy: General versus specific risk factors. J Neurol Sci 331: 19-25.

Ben-Ari Y. 2002. Excitatory actions of GABA during development: The nature of the nurture. Nat Rev Neurosci 3: 728-739.

Berg AT, Millichap JJ. 2013. The 2010 revised classification of seizures and epilepsy. Continuum (Minneap Minn) 19: 571-597.

Berg AT, Shinnar S. 1991. The risk of seizure recurrence following a first unprovoked seizure: A quantitative review. Neurology 41: 965-967.

Berg AT, Berkovic SF, Brodie MJ, Buchhalter J, Cross JH, van Emde Boas W, Engel J, Glauser TA, Mathern GW, Moshe SL, et al. 2010. Revised terminology and concepts for organization of seizures and epilepsy: Report of the 
C.E. Stafstrom and L. Carmant

ILAE Commission on Classification and Terminology, 2005-2009. Epilepsia 51: 676-685.

* Berkovic SF. 2015. Genetics of epilepsy in humans. Cold Spring Harb Perspect Med doi: 10.1101/cshperspect .a022400.

Bernhardt BC, Hong S, Bernasconi A, Bernasconi N. 2013. Imaging structural and functional brain networks in temporal lobe epilepsy. Front Hum Neurosci 7: 624.

Brooks-Kayal AR, Bath KG, Berg AT, Galanopoulou AS, Holmes GL, Jensen FE, Kanner AM, O’Brien TJ, Whittemore $\mathrm{VH}$, Winawer MR, et al. 2013. Issues related to symptomatic and disease-modifying treatments affecting cognitive and neuropsychiatric comorbidities of epilepsy. Epilepsia 54: 44-60.

Buckmaster PS. 2004. Laboratory animal models of temporal lobe epilepsy. Comp Med 54: 473-485.

* Bui A, Kim H, Moroso M, Soltesz I. 2015. Microcircuits in epilepsy: Heterogeneity and hub cells in network synchronization. Cold Spring Harb Perspect Med doi: 10.1101/cshperspect.a022855.

Cain SM, Snutch TP. 2013. T-type calcium channels in burst-firing, network synchrony, and epilepsy. Biochim Biophys Acta 1828: 1572-1578.

Camfield C, Camfield P, Gordon K, Dooley J. 1996. Does the number of seizures before treatment influence ease of control or remission of childhood epilepsy? Not if the number is 10 or less. Neurology 37: 19-23.

Caruso PA, Johnson J, Thibert R, Rapalino O, Rincon S, Ratai EM. 2013. The use of magnetic resonance spectroscopy in the evaluation of epilepsy. Neuroimaging Clin N Am 23: 407-424.

Chadwick D, Taylor J, Johnson T. 1996. Outcomes after seizure recurrence in people with well-controlled epilepsy and the factors that influence it. The MRC Antiepileptic Drug Withdrawal Group. Epilepsia 37: 1043-1050.

Cornejo BJ, Mesches MH, Coultrap S, Browning MD, Benke TA. 2007. A single episode of neonatal seizures permanently alters glutamatergic synapses. Ann Neurol 61: 411-426.

Cortez MA, McKerlie C, Snead OC. 2001. A model of atypical absence seizures: EEG, pharmacology, and developmental characterization. Neurology 56: 341-349.

* Coulter DA, Steinhäuser C. 2015. Role of astrocytes in epilepsy. Cold Spring Harb Perspect Med doi: 10.1101/ cshperspect.a022434.

Coulter DA, Huguenard JR, Prince DA. 1989. Specific petit mal anticonvulsants reduce calcium currents in thalamic neurons. Neurosci Lett 98: 74-78.

Davis R, Dalmau J. 2013. Autoimmunity, seizures, and status epilepticus. Epilepsia 54: 46-49.

Dravet C, Bureau M, Oguni H, Fukuyama Y, Cokar O. 2005. Severe myoclonic epilepsy in infancy: Dravet syndrome. Adv Neurol 95: 71-102.

Duchowny M, Cross JH, Arzimanoglou A. 2012. Pediatric epilepsy. McGraw-Hill, New York.

Dudek FE, Sutula TP. 2007. Epileptogenesis in the dentate gyrus: A critical perspective. Prog Brain Res 163: 755773.

Engel J Jr. 2013. Seizures and epilepsy. Oxford University Press, Oxford.
Engel J Jr, Pedley TA. (ed.). 2008. Epilepsy: A comprehensive textbook. Wolters Kluwer, Philadelphia.

Escayg A, Goldin AL. 2010. Sodium channel SCN1A and epilepsy: Mutations and mechanisms. Epilepsia 51: 1650-1658.

Escayg A, MacDonald BT, Meisler MH, Baulac S, Huberfeld G, Gourfinkel-An I, Brice I, LeGuern E, Moulard B, Chaigne D, et al. 2000. Mutations of SCN1A, encoding a neuronal sodium channel, in two families with $\mathrm{GEFS}^{+2}$. Nat Genet 24: 343-345.

Fisher RS. 2009. What clinicians want to know from epilepsy researchers. Epilepsia 50: 364-367.

Glass HC, Wusthoff CJ, Shellhaas RA. 2013. Amplitudeintegrated electro-encephalography: The child neurologist's perspective. J Child Neurol 28: 1342-1350.

Glauser T, Ben-Menachem E, Bourgeois B, Cnaan A, Guerreiro C, Kälviäinen R, Mattson R, French JA, Perucca E, Tomson T, et al. 2013. Updated ILAE evidence review of antiepileptic drug efficacy and effectiveness as initial monotherapy for epileptic seizures and syndromes. Epilepsia 54: 551-563.

Hancock EC, Cross JH. 2013. Treatment of Lennox-Gastaut syndrome. Cochrane Database Syst Rev 2: CD003277.

Hartman AL, Cross JH. 2014. Timing of surgery in Rasmussen syndrome: Is patience a virtue? Epilepsy Curr 14: 8 11.

Hauser W, Hersdorffer D. 1990. Epilepsy: Frequency, causes and consequences. Demos, New York.

Hauser WA, Rich SS, Lee JR, Annegers JF, Anderson VE. 1998. Risk of recurrent seizures after two unprovoked seizures. N Engl J Med 338: 429-434.

* Holmes GL. 2015. The neurobiology of epilepsy: Future directions. Cold Spring Harb Perspect Med doi: 10.1101/ cshperspect.a022749.

Holmes G, Ben-Ari Y. 1998. Seizures in the developing brain: Perhaps not so benign after all. Neuron 21: 1231-1234.

Issa NP. 2014. Neurobiology of continuous spike-wave in slow-wave sleep and Landau-Kleffner syndromes. Pediatr Neurol 51: 287-296.

Joshi S, Rajasekaran K, Kapur J. 2013. GABAergic transmission in temporal lobe epilepsy: The role of neurosteroids. Exp Neurol 244: 36-42.

Kanner AM. 2013. The treatment of depressive disorders in epilepsy: What all neurologists should know. Epilepsia 54: $3-12$.

Kay B, Szaflarski JP. 2014. EEG/fMRI contributions to our understanding of genetic generalized epilepsies. Epilepsy Behav 34: 129-135.

Kim H, Paige AL, Knowlton RC. 2010. Advances in structural and functional neuroimaging: How are these guiding epilepsy surgery? In Epilepsy: Mechanisms, models, and translational perspectives (ed. Rho JM, Sankar R, Stafstrom CE), pp. 257-282. CRC, Boca Raton, FL.

Lado FA, Rubboli G, Capovilla P, Avanzini G, Moshé SL. 2013. Pathophysiology of epileptic encephalopathies. Epilepsia 54: 6-13.

LaFrance WC Jr, Reuber M, Goldstein LH. 2013. Management of psychogenic nonepileptic seizures. Epilepsia 54: $53-67$. 
Landau WM, Kleffner FR. 1957. Syndrome of acquired aphasia with convulsive disorder in children. Neurology 7: $523-530$.

* Lenck-Santini P-P, Scott R. 2015. Mechanisms responsible for cognitive impairment in epilepsy. Cold Spring Harb Perspect Med doi: 10.1101/cshperspect.a022772.

Lewis DV, Shinnar S, Hesdorffer DC, Bagiella E, Bello JA, Chan S, Xu Y, MacFall J, Gomes WA, Moshé SL, et al. 2014. Hippocampal sclerosis after febrile status epilepticus: The FEBSTAT study. Ann Neurol 75: 178-185.

Liu Z, Mikati M, Holmes GL. 1995. Mesial temporal sclerosis: Pathogenesis and significance. Pediatr Neurol 12: 5-16.

Liu Y, Lopez-Santiago LF, Yuan Y, Jones JM, Zhang H, O’Malley HA, Patino GA, O’Brien JE, Rusconi R, Gupta A, et al. 2013. Dravet syndrome patient-derived neurons suggest a novel epilepsy mechanism. Ann Neurol 74: 128 139.

Lortie A. 2013. Psychogenic nonepileptic seizures. Handb Clin Neurol 112: 875-879.

Löscher W, Puskarjov M, Kaila K. 2013. Cation-chloride cotransporters NKCC1 and KCC2 as potential targets for novel antiepileptic and antiepileptogenic treatments. Neuropharmacology 69: 62-74.

Macdonald RL, Kang JQ, Gallagher MJ. 2010. Mutations in $\mathrm{GABA}_{\mathrm{A}}$ receptor subunits associated with genetic epilepsies. J Physiol 588: $1861-1869$.

McClelland S, Dubé CM, Yang J, Baram TZ. 2011. Epileptogenesis after prolonged febrile seizures: Mechanisms, biomarkers and therapeutic opportunities. Neurosci Lett 497: 155-162.

Michelucci R, Pasini E, Riguzzi P, Volpi L, Dazzo E, Nobile C. 2012. Genetics of epilepsy and relevance to current practice. Curr Neurol Neurosci Rep 12: 445-455.

Miya K, Takahashi Y, Mori H. 2014. Anti-NMDAR autoimmune encephalitis. Brain Dev 36: 645-652.

Mullen SA, Carvill GL, Bellows S, Bayly MA, Trucks H, Lal D, Sander T, Berkovic SF, Dibbens LM, Scheffer IE, et al. 2013. Copy number variants are frequent in genetic generalized epilepsy with intellectual disability. Neurology 81: 1507-1514.

Muro VM, Connolly MB. 2014. Classifying epileptic seizures and the epilepsies. In Epilepsy (ed. Miller JW, Goodkin HP), pp. 10-14. Wiley, Chichester, UK.

Nabbout R, Dulac O. 2008. Epileptic syndromes in infancy and childhood. Curr Opin Neurol 21: 161-166.

Neligan A, Bell GS, Elsayed M, Sander JW, Shorvon SD. 2012. Treatment changes in a cohort of people with apparently drug-resistant epilepsy: An extended follow-up. J Neurol Neurosurg Psychiatry 83: 810-813.

Oakley JC, Kalume F, Catterall WA. 2011. Insights into pathophysiology and therapy from a mouse model of Dravet syndrome. Epilepsia 52: 59-61.

Obeid M, Mikati MA. 2007. Expanding spectrum of paroxysmal events in children: Potential mimickers of epilepsy Pediatr Neurol 37: 309-316.

Olson HE, Poduri A. 2014. Epilepsy: When to perform a genetic analysis. In Epilepsy (ed. Miller JW, Goodkin HP), pp. 159-166. Wiley, Chichester, UK.

Olson H, Shen Y, Avallone J, Sheidley BR, Pinsky R, Bergin AM, Berry GT, Duffy FH, Eksioglu Y, Harris DJ, et al.
2014. Copy number variation plays an important role in clinical epilepsy. Ann Neurol 75: 943-958.

Painter MJ, Scher MS, Stein AD, Armatti S, Wang Z, Gardner JC, Paneth N, Minnigh B, Alvin J. 1999. Phenobarbital compared with phenytoin for the treatment of neonatal seizures. N Engl J Med 341: 485-489.

Patterson KP, Baram TZ, Shinnar S. 2014. Origins of temporal lobe epilepsy: Febrile seizures and febrile status epilepticus. Neurotherapeutics 11: 242-250.

Pavlidou E, Panteliadis C. 2013. Prognostic factors for subsequent epilepsy in children with febrile seizures. Epilepsia 54: 2101-2107.

Pearl PL. 2009. New treatment paradigms in neonatal metabolic epilepsies. J Inherit Metab Dis 32: 204-213.

Pearl PL, Taylor JL, Trzcinski S, Sokohl A. 2007. The pediatric neurotransmitter disorders. J Child Neurol 22: 606616.

Pearson TS, Akman C, Hinton VJ, Engelstad K, DeVivo DC. 2013. Phenotypic spectrum of glucose transporter type 1 deficiency syndrome (Glut1 DS). Curr Neurol Neurosci Rep 13: 342.

* Pitkänen A, Lukasiuk K, Dudek FE, Staley KJ. 2015. Epileptogenesis. Cold Spring Harb Perspect Med doi: 10.1101/ cshperspect.a022822.

Poduri A, Lowenstein D. 2011. Epilepsy genetics-Past, present, and future. Curr Opin Genet Dev 21: 325-332.

Rogawski MA, Bazil CW. 2008. New molecular targets for antiepileptic drugs: $\alpha 2 \sigma$, SV2A, and $\mathrm{Kv} 7 / \mathrm{KCNQ} / \mathrm{M}$ potassium channels. Curr Neurol Neurosci Rep 8: 345-352.

Schmidt D, Löscher W. 2005. Uncontrolled epilepsy following discontinuation of antiepileptic drugs in seizure-free patients: A review of current clinical experience. Acta Neurol Scand 111: 291-300.

Schuchmann S, Schmitz D, Rivera C, Vanhatalo S, Salmen B, Mackie K, Sipilä ST, Voipio J, Kaila K. 2006. Experimental febrile seizures are precipitated by a hyperthermia-induced respiratory alkalosis. Nat Med 12: $817-$ 823.

Shinnar S, Berg AT, O’Dell C, Newstein D, Moshe SL, Hauser WA. 2000. Predictors of multiple seizures in a cohort of children prospectively followed from the time of their first unprovoked seizure. Ann Neurol 48: 140-147.

Shirley MD, Tang H, Gallione CJ, Baugher JD, Frelin LP, Cohen B, North PE, Marchuk DA, Comi AM, Pevsner J. 2013. Sturge-Weber syndrome and port-wine stains caused by somatic mutation in GNAQ. $N$ Engl J Med 368: 1971-1979.

Shorvon SD, Andermann F, Guerrini R. (ed.). 2011. The causes of epilepsy. Cambridge University Press, Cambridge.

Sillanpää M, Schmidt D. 2009. Early seizure frequency and aetiology predict long-term medical outcome in childhood-onset epilepsy. Brain 132: 989-998.

Stafstrom CE. 2009. Infantile spasms: A critical review of emerging animal models. Epilepsy Curr 9: 75-81.

Stafstrom CE. 2010. Pathophysiological mechanisms of seizures and epilepsy: A primer. In Epilepsy: Mechanisms, models, and translational perspectives (ed. Rho JM, Sankar R, Stafstrom CE), pp. 3-19. CRC, Boca Raton, FL. 


\section{C.E. Stafstrom and L. Carmant}

Stafstrom CE, Arnason BGW, Baram TZ, Catania A, Cortez MA, Glauser TA, Pranzatelli MR, Riikonen R, Rogawski MA, Shinnar S, et al. 2011. Treatment of infantile spasms: Emerging insights from clinical and basic science perspectives. J Child Neurol 26: 1411-1421.

Staley KJ. 2006. Role of the depolarizing GABA response in epilepsy. Adv Exp Med Biol 548: 104-109.

Steinlein OK. 2014. Mechanisms underlying epilepsies associated with sodium channel mutations. Prog Brain Res 213: $97-111$.

Steinlein OK, Conrad C, Weidner B. 2007. Benign familial neonatal convulsions: Always benign? Epilepsy Res 73: 245-249.

Strine TW, Kobau R, Chapman DP, Thurman DJ, Price P, Balluz LS. 2005. Psychological distress, comorbidities, and health behaviors among U.S. adults with seizures: Results from the 2002 National Health Interview Survey. Epilepsia 46: 1133-1139.

Surges R, Sander JW. 2014. Sudden unexpected death in epilepsy: Mechanisms, prevalence, and prevention. Curr Opin Neurol 25: 201-207.

Swann JW, Moshe SL. 2012. On the basic mechanisms of infantile spasms. In Jasper's basic mechanisms of the epilepsies (ed. Noebels JL, Avoli M, Rogawski MA, Olsen RW, Delgado-Escueta AV), pp. 272-285. Oxford University Press, Oxford.
Thom M, Mathern GW, Cross JH, Bertram EH. 2010. Mesial temporal lobe epilepsy: How do we improve surgical outcome? Ann Neurol 68: 424-434.

Thomas RH, Berkovic SF. 2014. The hidden genetics of epilepsy-A clinically important new paradigm. Nat Rev Neurol 10: 283-292.

Van Bogaert P. 2013. Epileptic encephalopathy with continuous spike-waves during slow-wave sleep including Landau-Kleffner syndrome. Handb Clin Neurol 111: 635640.

Varadkar S, Bien CG, Kruse CA, Jensen FE, Bauer J, Pardo CA, Vincent A, Mathern GW, Cross JH. 2014. Rasmussen's encephalitis: Clinical features, pathobiology, and treatment advances. Lancet Neurol 13: 195-205.

Verrotti A, Beccaria F, Fiori F, Montagnini A, Capovilla G. 2012. Photosensitivity: Epidemiology, genetics, clinical manifestations, assessment, and management. Epileptic Disord 14: 349-362.

* Vezzani A, Lang B, Aronica E. 2015. Immunity and inflammation in epilepsy. Cold Spring Harb Perspect Med doi: 10.1101/cshperspect.a022699.

Yu FH, Mantegazza M, Westenbroek RE, Robbins CA, Kalume F, Burton KA, Spain WJ, McKnight GS, Scheuer T, Catterall WA. 2006. Reduced sodium current in GABAergic interneurons in a mouse model of severe myoclonic epilepsy in infancy. Nat Neurosci 9: 11421149. 


\section{$\& \mathrm{CSH} \&$ Cold Spring Harbor

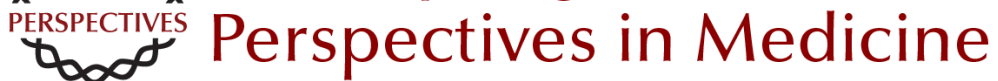

\section{Seizures and Epilepsy: An Overview for Neuroscientists}

Carl E. Stafstrom and Lionel Carmant

Cold Spring Harb Perspect Med 2015; doi: 10.1101/cshperspect.a022426

Subject Collection Epilepsy: The Biology of a Spectrum Disorder

The Epilepsy Spectrum: Targeting Future Research Challenges

Gregory L. Holmes and Jeffrey L. Noebels

Role of Sodium Channels in Epilepsy

David I. Kaplan, Lori L. Isom and Steven Petrou

\section{Mechanisms of Action of Antiseizure Drugs and the Ketogenic Diet Michael A. Rogawski, Wolfgang Löscher and Jong M. Rho}

Epilepsy and Autism

Ashura W. Buckley and Gregory L. Holmes

Immunity and Inflammation in Epilepsy Annamaria Vezzani, Bethan Lang and Eleonora Aronica

Hyperpolarization-Activated Cyclic Nucleotide-Gated (HCN) Channels in Epilepsy Gary P. Brennan, Tallie Z. Baram and Nicholas P. Poolos

The Role of Calcium Channels in Epilepsy Sanjeev Rajakulendran and Michael G. Hanna Interneuron Transplantation as a Treatment for Epilepsy

Robert F. Hunt and Scott C. Baraban
Common Mechanisms Underlying

Epileptogenesis and the Comorbidities of

Epilepsy

Andrey Mazarati and Raman Sankar

The Diathesis-Epilepsy Model: How Past Events Impact the Development of Epilepsy and Comorbidities Christophe Bernard

Potassium Channels in Epilepsy Rüdiger Köhling and Jakob Wolfart

\section{GABAergic Synchronization in Epilepsy} Roustem Khazipov

\section{Status Epilepticus}

Syndi Seinfeld, Howard P. Goodkin and Shlomo Shinnar

Neonatal and Infantile Epilepsy: Acquired and Genetic Models Aristea S. Galanopoulou and Solomon L. Moshé

Epigenetics and Epilepsy David C. Henshall and Katja Kobow

Microcircuits in Epilepsy: Heterogeneity and Hub Cells in Network Synchronization Anh Bui, Hannah K. Kim, Mattia Maroso, et al.

For additional articles in this collection, see http://perspectivesinmedicine.cshlp.org/cgi/collection/ 